\title{
DNA mismatch repair in advanced metastatic prostate carcinoma: clinical perspective
}

\begin{abstract}
Prostate carcinoma ( $\mathrm{PCa}$ ) is among the most frequently diagnosed cancer in men worldwide. Men with metastatic PCa receive primary androgen deprivation therapy (ADT). However, nearly all men will develop resistance to primary ADT, a state known as castration-resistant prostate carcinoma a (CRPC). Several therapeutic drugs with different mechanisms of action have been approved for CRPC including systemic chemotherapeutics (docetaxel and cabazitaxel) and drugs targeting the resistance pathways leading to CRPC, including enzalutamide and abiraterone. Despite significant survival benefit, resistance to these therapies develops rapidly. Thus, deciphering the mechanisms of resistance and pathways leading to progression is the most critical objectives in PCa research. Some proofof-concept clinical trials have identified that personalized therapies with PARP inhibition, platinum chemotherapy, and immunotherapy may be beneficial to a subset of PCa and CRCP with underlying DNA repair defects. This review aims to address the potential therapeutic implication of Mismatch repair (MMR) pathways in advanced PCa and CRPC.
\end{abstract}

Volume 8 Issue 7 - 2017

\author{
Abha Soni, Anju Bansal \\ ICMR-National Institute of Pathology, Safdarjung Hospital \\ Campus, India
}

Correspondence: Dr.Anju Bansal, Scientist E (Deputy Director), ICMR-National Institute of Pathology, Safdarjung Hospital Campus, Post Box No. 4909, New Delhi - 110029 India, Tel +9I-98I0368354, Fax +9I-0I I-26I98 40 I Email abhasoni.iop@gmail.com

Received: November 27, 2017 | Published: December II, 2017

Keywords: Prostate cancer; CRPC; MMR; PARP

Abbreviations: $\mathrm{PCa}$, prostate carcinoma; CRPC, castrationresistant prostate carcinoma; MMR, mismatch repair; ADT, androgendeprivation therapy; OS, overall survival; NER, nucleotide excision repair; BER, base excision repair; HDR, homology directed repair; HR, homologous recombination; DDR, DNA damage response; NHEJ, non-homologous end joining; SSB, single strand breaks; PARP, poly (adenosine diphosphate. ${ }^{A D P}$-ribose) polymerase; PARPi: PARP inhibitors

\section{Introduction}

Prostate carcinoma a $(\mathrm{PCa})$ is the second most frequently diagnosed cancer in men worldwide, constituting $15 \%$ of the total new cases diagnosed in 2012. ${ }^{1} \mathrm{PCa}$ is primarily a disease of elderly men and its incidence trend increases in the seventh decade of life. Nevertheless, it is a highly heterogeneous disease, characterized by variance in histology, genomic, and molecular characteristics, and response to therapy. For locally advanced and metastatic PCa, the most frequent therapeutic option is suppressing the tumor growth by androgen-deprivation therapy (ADT). The majority of cases with $\mathrm{PCa}$ responds to initial therapy and achieve a long-term remission; however, development of advanced castration-resistant PCa (CRPC) is inevitable. ${ }^{3,4}$ Several drugs with different therapeutic mechanisms of action have been shown to prolong overall survival (OS) in patients with CRPC. These include the tubulin targeting chemotherapies such as docetaxel and cabazitaxel, immunotherapeutics like sipuleucel-T, inhibitors of the androgen biosynthesis abiraterone, the second generation androgen receptor (AR) antagonist enzalutamide and the alpha-emitting radiopharmaceutical Radium-223. 5,6 However, the development of resistance to these drugs and cross-resistance remains a clinically relevant concern. Despite the extensive understanding of the molecular events underlying the progression of prostate tumorigenesis, the molecular mechanisms leading to therapy resistance and relapse remain limited.

Over the recent years, platinum compounds have been investigated in some clinical trials in $\mathrm{PCa}$ patients with varying molecular characteristics. Moreover, molecular profiling of advanced CRPC has shown that a significant proportion of patients harbor defects in DNA mismatch repair genes, which may serve as prognostic markers for sensitivity to platinum agents. ${ }^{7}$ Recently, poly (adenosine diphosphate ADP-ribose) polymerase (PARP) enzyme received increased attention as a potential target for chemotherapeutics, as inhibition of PARP leads to a significant DNA damage through double-strand breaks (DSBs) by interfering with the replication fork. Thus, PARP inhibitors (PARPi) can efficiently target those cells with damaged DNA. It has also been proposed that inhibition of PARP1 could further be enhanced by applying a synthetic lethal approach. According to this concept, two mutations occurring within either tumor suppressors or proto-oncogenes might result in cell death. Moreover, a clinical trial TOPARP has shown the remarkable antitumor activity of the PARPi, olaparib in patients with advanced CRPC, confirming that targeting DNA repair defects has a therapeutic potential in specific subsets of patients. ${ }^{8}$ Therefore, the present study aims to review the therapeutic significance of DNA repair, particularly DNA mismatch repair in advanced PCa and CRPC. Further, mechanisms of resistance to chemotherapy will also be briefly discussed.

\section{DNA mismatch repair system}

Mismatch repair (MMR) system recognizes non-WatsonCrick base pairs and strand mismatches generated during DNA replication and mediate their elimination by catalyzing excision of the mispair-containing region of nascent DNA. ${ }^{9}$ Thus, MMR act as the in-charge of DNA repair by promoting genetic stability following DNA polymerase errors, preventing recombination between nonidentical DNA sequences and contributing in responses to DNA damage. ${ }^{10}$ MMR also recognizes DNA damage generated by chemical compounds, such as alkylating agents.

Nevertheless, MMR proteins interact with each other to form protein heterodimers, MutL homolog 1 (MLH1) complexes with postmeiotic segregation increased 1 (PMS1), or PMS2 or MLH3 to give rise to MLH1/PMS2 (MutL $\alpha)$, or MLH1/PMS1(MutL $\beta)$, heterodimers. However, MutS homolog 2 (MSH2) complexes with MSH6 or MSH3 to generate heterodimers of the MSH2/ MSH6 (MutS $\alpha$ ) or MSH2/MSH3 (MutS $\beta) .{ }^{11}$ These complexes then help in recognition and digestion of mismatched DNA segments and create error-free DNA helix. 
Besides MMR other important DNA repair pathways include base excision repair (BER), nucleotide excision repair (NER), and double-strand break repair (DDR), which play a significant role in DNA repair. Notably, DNA damage responses, and predominantly DNA repair, influence the outcome of therapy. Moreover, a substantial association of DNA repairs and drug sensitivity has been demonstrated using cell lines. ${ }^{12}$ This indicates a more complex relationship between the efficiency of DNA repair mechanism and tumor therapy. Furthermore, approximately $10 \%$ of advanced $\mathrm{PCa}$ have a significantly elevated rate of single nucleotide mutations. This has been associated with underlying somatic and germline defects in MMR family constituents ( $h M S H 2, h M S H 6, h M S H 3, h M L H 1$, $h P M S 1$ or $h P M S 2$ ) and is characterized by microsatellite instability (MSI). Besides, inactivation of MMR genes has been shown to predict sensitivity to immunotherapy in metastatic $\mathrm{PC}$, similar to that seen in colorectal carcinoma (CRC)..$^{13,14}$

\section{Therapeutic significance of DNA mismatch repair in prostate carcinoma}

Deficiency of one of the MMR proteins has been associated with an increased predisposition to some malignancies including CRC, gastric, endometrial and ovarian, brain, and skin. ${ }^{15}$ The defects in MMR genes may be attributed to mutation, or methylation or a combination of the two. Furthermore, $20 \%-25 \%$ of advanced $\mathrm{PCa}$, exhibited germline or somatic mutations in $B R C A 1, B R C A 2$, or other genes involved in the high-fidelity homologous recombination (HR) pathway of DNA repair as revealed by genomic analysis. More recently, a synthetic lethal therapeutic approach using PARP inhibitor therapy has been developed for $B R C A$ mutant negative and HR deficient-related cancers (those with "BRCAness"). ${ }^{16}$ And it is also suggested that defective HR secondary to BRCA1 and BRCA2 mutations may render cancer cells particularly sensitive to inhibition of singles stranded binding proteins (SSB) repair through inhibition of PARP. ${ }^{17}$ Particularly, PARP inhibitors such as olaparib and niraparib exhibited significant antitumor efficacy in ovarian, breast, and $\mathrm{PCa}$ with dysfunctional HR. ${ }^{18}$ Moreover, these are being extensively investigated in multiple clinical trials. PARP inhibitors are undergoing trials in different tumor types including ovarian, breast, pancreatic, gastric, non-small cell lung cancer, melanoma, glioblastoma, and other cancers. ${ }^{19}$

Studies have demonstrated that men younger than 65 years who are $B R C A 1$ mutation carriers are more susceptible to $\mathrm{PCa}$ as compared to those who are not the carriers of the mutation. ${ }^{20}$ Recently, Fong et $a l$. reported that olaparib exhibited potential antitumor activity only in patients with BRCA1 or BRCA2 mutations..$^{21}$ Moreover, Mateo et al demonstrated that three of four patients with a $B R C A 2$ mutation and an $E R G$ rearrangement exhibited a significant response to olaparib (10-34 months on treatment), while the fourth patient exhibited primary resistance to PARP inhibitor MK-4827. ${ }^{8}$ Taken together, PARP inhibitors are mainly useful in tumors with HR deficiency; however, further studies are required to identify novel biomarkers (in addition to BRCA1 or BRCA2) to stratify patients who would be most benefitted from this therapy.

The concept of synthetic lethality is inherently plausible in rare familial PCa, AR-mediated regulation of DNA Damage Response (DDR) genes may provide an understanding of synergistic combinations by employing novel inhibitors of androgen synthesis and anti-androgens including abiraterone and enzalutamide, to inhibit DDR..$^{22}$ More specifically, inhibition of AR leads to downregulation of DDR function to generate a defect in DNA damage repair. Thus, targeting AR-inhibited tumors with a drug that induces damage to
DNA, synthetic lethality or synergistic cytotoxicity may be achieved. Moreover, in preclinical models, ADT combined with a PARP inhibitor has demonstrated encouraging results. ${ }^{22}$ Currently, an ongoing clinical trial (ClinicalTrials.gov ID NCT01576172) in patients with mCRPC, where patients were stratified based on their ETS gene fusion status (positive or negative) and then randomised to administer abiraterone alone or combination of abiraterone and velaparib. This trial is expected to shed light on the potential synergistic effects of combined ADT and PARP inhibition.

Furthermore, to study the mechanism of resistance to PARP inhibitor, researchers designed PARP-inhibitor-resistant (PIR) cells to identify the molecular kinetics of PAPRi including screening of RNA inference (RNAi), and knockin mouse models. Tumor cells may exhibit resistance to this therapy through overexpression of PARP1, increased the drugs efflux, prevention of inhibitors' binding to PARP1, restoration of HR repair pathway, and suppression of nonhomologous end joining pathway. ${ }^{23}$ Comprehensive studies involving both in vitro and in vivo study designs are undergoing to elucidate the mechanisms of resistance to PAPRi completely. Notably, an in vivo model demonstrated that cancer cells treated with Olaparib drug develop resistance through the partial restoration of HR DNA repair pathway. $^{24}$

Anticancer drugs are significantly influenced by the cellular DNA repair capacity, and specific changes in DNA MMR pathways have been reported to be associated with differences in patient response to chemotherapy in several cancers. ${ }^{25}$ MMR deficient tumors are resistant to a wide range of chemotherapeutic agents including methylating agents, platinum compounds, and fluoropyrimidine agents. A potential resistance to 5 -fluorouracil chemotherapy has also been described with mutations in MMR genes. ${ }^{26}$ Recently, a small molecule, baicalein was also identified as a novel chemotherapeutic agent that selectively targets MutSa-deficient cancer cells. ${ }^{27}$ Nevertheless, genetic polymorphisms in the MMR pathway have also been predicted to serve as prognostics clinical markers in predicting responses to chemotherapy in patients with lung cancer. Furthermore, two randomized clinical trials on a pooled multivariable analysis revealed that deficient vs proficient MMR repair status was significantly correlated with more prolonged disease-free survival in patients with stage III colon cancer who received FOLFOX alone. ${ }^{28}$

Extensive research in various cancers has revealed multiple mechanisms that underlie recognition and MMR repair of DNA damage induced by platinum-based agents. A better understanding of these mechanisms has become crucial because virtually all $\mathrm{PCa}$ and CRPC cancers become resistant to this type of chemotherapy. Moreover, the cellular DNA MMR responses to platinum-based agentinduced DNA damage cannot be predicted due to the complexity of these mechanisms, and the alkylation damage may induce a variety of repair mechanisms. Taken together, future studies in the adjuvant setting should consider the status of MMR as an essential stratification factor.

\section{Personalized treatment care in $\mathbf{P C a}$}

The interpatient molecular heterogeneity of $\mathrm{PCa}$ is well recognized; however, treatment to date has not been stratified by molecular mechanisms. Further, it is also believed that MMR inactivation is not particularly associated with intrinsic resistance in advanced Pca and CRPC. ${ }^{27}$ However, MMR inactivation might play a crucial role in acquired resistance because maybe MMR deficient cells are selectively targeted during platinum-based chemotherapy, this needs further investigation. 
Furthermore, genomic aberrations in the DNA repair are common in $\mathrm{PCa}$ and occur in about $20-30 \%$ of advanced PCa patients. Some of these genetic aberrations have been associated with sensitivity to platinum and PARP inhibitors in preclinical studies. ${ }^{29}$ As PARP is involved in multiple aspects of DNA repair, PARPi, olaparib has been approved for treating ovarian cancers with $B R C A 1 / 2$ mutations. ${ }^{30}$ PARP inhibition exhibited significant antitumor activity in men with advanced metastatic CRPC, and deleterious germline mutations in BRCA2. Studies have also demonstrated that olaparib would have antitumor activity in sporadic cases of metastatic, CRPC with DNArepair defects. ${ }^{12}$

Given the marked heterogeneity in biological behaviour of PCa, it is desirable that the treatment is personalized based on the molecular mechanisms. Mainly, the need of the hour is to develop patientspecific therapies with drugs for antiproliferative and immunotherapy approaches targeting the susceptible points in $\mathrm{PCa}$ and advanced metastatic CRPC. It is therefore imperative to develop novel strategies to reduce or overcome chemoresistance in cancer. Within this context, men with metastatic CRPC and DNA-repair gene mutations have been reported to exhibit sustained responses to PARPi and platinumbased chemotherapy. As stated earlier, Phase II trial of TOPARP-A has indicated that olaparib exhibited significant antitumor activity in a subset of cases of metastatic CRPC characterized by defects in DNA repair.

\section{Conclusion}

In conclusion, for more precise, personalized care in $\mathrm{PCa}$ and CRPC, a novel classification based on molecular characteristics relevant to prognosis and treatment is desirable. For patients identified with DNAMMR mutations, studies must be conducted to determine the best clinicopathological status (localized PCa vs. advanced metastatic castration-sensitive PCa vs. metastatic CRPC) to introduce PARP inhibitors or DNA-damaging chemotherapy/immunotherapy. Beyond platinum-based and PARP inhibitors, other DNA damaging agents also need to be evaluated, including anthracyclines and topoisomerase inhibitors. It is uncertain at this point if genomic sequencing should be analyzed in primary prostate biopsy or prostatectomy specimens, or if should this be reserved for the patients with refractory metastatic disease progressing past abiraterone, enzalutamide, and chemotherapy.

\section{Acknowledgements}

We thank Indian Council of Medical Research for providing necessary funds to support this study.

\section{Conflicts of Interest}

None of the authors have any conflicts of interest.

\section{Funding}

None.

\section{References}

1. Torre LA, Bray F, Siegel RL, et al. Global Cancer Statistics. CA a cancer J Clin. 2015;65(2):87-108.

2. Barqawi A, Crawford ED. Testosterone replacement therapy and the risk of prostate cancer. Is there a link? Int J Impot Res. 2006;18(4):323-328.

3. Chandrasekar T, Yang JC, Gao AC, et al. Mechanisms of resistance in castration-resistant prostate cancer (CRPC). Transl Androl Urol. 2015;4(3):365-380.
4. Yuan X, Cai C, Chen S, et al. Androgen receptor functions in castrationresistant prostate cancer and mechanisms of resistance to new agents targeting the androgen axis. Oncogene. 2014;33(22):2815-2825.

5. Imamura Y, Sadar MD. Androgen receptor targeted therapies in castration-resistant prostate cancer: Bench to clinic. International Journal of Urology. 2016;23:65465.

6. Omlin A, Pezaro C, Gillessen Sommer S. Sequential use of novel therapeutics in advanced prostate cancer following docetaxel chemotherapy. Ther Adv Urol. 2014;6(1):3-14.

7. Hager S, Ackermann CJ, Joerger M, et al. Anti-tumour activity of platinum compounds in advanced prostate cancer-a systematic literature review. Ann Oncol. 2016;7(6):975-984.

8. Mateo J, Carreira S, Sandhu S, et al. DNA-Repair Defects and Olaparib in Metastatic Prostate Cancer. N Engl J Med. 2015;373(18):1697-1708.

9. Jiricny J. Postreplicative mismatch repair. Cold Spring Harbor Perspectives in Biology. 2013;5(4):a012633.

10. Harfe BD, Jinks-Robertson S. DNA mismatch repair and genetic instability. Annu Rev Genet. 2000;34:359-399.

11. Matton N, Simonetti J, Williams K. Identification of mismatch repair protein complexes in HeLa nuclear extracts and their interaction with heteroduplex DNA. J Biol Chem. 2000;275(23):17808-17813.

12. Mateo J, Boysen G, Barbieri CE, et al. DNA Repair in Prostate Cancer: Biology and Clinical Implications. Eur Urol. 2016;71(3):417-425.

13. Guedes L, Antonarakis ES, Schweizer MT, et al. MSH2 Loss in Primary Prostate Cancer. Clin Cancer Res. 2017;23(22):6863-6874.

14. Bupathi $\mathrm{M}, \mathrm{Wu} \mathrm{C}$. Biomarkers for immune therapy in colorectal cancer: mismatch-repair deficiency and others. $J$ Gastrointest Oncol.2016;7(5):713-720.

15. Valle L. Genetic predisposition to colorectal cancer: Where we stand and future perspectives. World J Gastroenterol. 2014;20(29):9828-9849.

16. Dhawan M, Ryan CJ, Ashworth A. DNA Repair Deficiency Is Common in Advanced Prostate Cancer: New Therapeutic Opportunities. Oncologist. 2016;21(8):940-945.

17. Gavande NS, Vandervere-Carozza PS, Hinshaw HD, et al. DNA repair targeted therapy: The past or future of cancer treatment? Pharmacology and Therapeutics. 2016;160:65-83.

18. Dziadkowiec KN, Gąsiorowska E, Nowak-Markwitz E, et al. PARP inhibitors: review of mechanisms of action and BRCA1/2 mutation targeting. Prz menopauzalny. 2016;15(4):215-279.

19. Cerrato A, Morra F, Celetti A. Use of poly ADP-ribose polymerase [PARP] inhibitors in cancer cells bearing DDR defects: the rationale for their inclusion in the clinic. J Exp Clin Cancer Res. 2016;35(1):179.

20. Friedenson B. BRCA1 and BRCA2 pathways and the risk of cancers other than breast or ovarian. Med Gen Med. 2005;7(2):60.

21. Audeh MW, Carmichael J, Penson RT, et al. Oral poly(ADP-ribose) polymerase inhibitor olaparib in patients with BRCA1 or BRCA2 mutations and recurrent ovarian cancer: A proof-of-concept trial. Lancet. 2010;376(9737):245-251.

22. Karanika S, Karantanos T, Li L, et al. DNA damage response and prostate cancer: defects, regulation and therapeutic implications. Oncogene. 2015;34(22):2815-2822.

23. Castro E, Mateo J, Olmos D, et al. Targeting DNA Repair: The Role of PARP Inhibition in the Treatment of Castration-Resistant Prostate Cancer. Cancer J. 2016;22(5):353-356.

24. Kim Y, Kim A, Sharip A, et al. Reverse the resistance to PARP inhibitors. Int J Biol Sci. 2017;13(2):198-208. 
25. Santarpia L, Iwamoto T, Di Leo A, et al. DNA repair gene patterns as prognostic and predictive factors in molecular breast cancer subtypes. Oncologist. 2013;18(10):1063-1073.

26. Sinicrope FA. DNA mismatch repair and adjuvant chemotherapy in sporadic colon cancer. Nat Rev Clin Oncol. 2010;7(3):174-177.

27. Zhang Y, Fox JT, Park YU, et al. A novel chemotherapeutic agent to treat tumors with DNA mismatch repair deficiencies. Cancer Res. 2016;76(14):4183-4191.

28. Zaanan A, Shi Q, Taieb J, et al. Role of Deficient DNA Mismatch Repair
Status in Patients With Stage III Colon Cancer Treated With FOLFOX Adjuvant Chemotherapy: A Pooled Analysis From 2 Randomized Clinical Trials. JAMA Oncol. 2017

29. Wadosky KM, Koochekpour S, Wadosky KM, et al. Molecular mechanisms underlying resistance to androgen deprivation therapy in prostate cancer. Oncotarget. 2016;7(39):64447-64470.

30. Konecny GE, Kristeleit RS. PARP inhibitors for BRCA1/2-mutated and sporadic ovarian cancer: current practice and future directions. $\mathrm{Br} J$ Cancer. 2016;115(10):1157-1173. 